\title{
Change of neuronal activations induced by the passive perception of driving speed difference
}

\author{
Hyun-Jun Kim ${ }^{\mathrm{a}}$, Jeong-Han Yi ${ }^{\mathrm{b}}$, Hyung-Sik Kim ${ }^{\mathrm{b}}$, Soon-Cheol Chung ${ }^{\mathrm{b}}$, Ji-Hye Baek ${ }^{\mathrm{b}}$, Jung- \\ Chul Lee ${ }^{\mathrm{b}}$, Sung-Jun Park ${ }^{\mathrm{b}}$, Ul-Ho Jeong, Seon-Young Gim ${ }^{\mathrm{b}}$, Sung-Phil Kim ${ }^{\mathrm{c}}$, Dae-Woon \\ $\mathrm{Lim}^{\mathrm{d}}$ and Mi-Hyun Choi ${ }^{\mathrm{b}, *}$ \\ ${ }^{a}$ Department of Obstetrics \& Gynecology, Konkuk University, Chungju, South Korea \\ ${ }^{b}$ Department of Biomedical Engineering, Research Institute of Biomedical Engineering, College of \\ Biomedical \& Health Science, Konkuk University, Chungju, South Korea \\ ${ }^{c}$ Department of Human and Systems Engineering, Ulsan National Institute of Science and Technology, \\ Ulsan, South Korea \\ ${ }^{d}$ Department of Information \& Communication Engineering, Dongguk University, Seoul, South Korea
}

\begin{abstract}
The change of neuronal activation due to the passive perception of various driving speeds in comparison to a reference driving speed was assessed using functional Magnetic Resonance Imaging. Videos recorded in real driving conditions on the road at driving speeds of $50,70,90$, and $110 \mathrm{~km} / \mathrm{h}$ were shown as visual stimuli. An experiment consisted of three blocks, each having a control phase $(50 \mathrm{~km} / \mathrm{h})$ and a stimulation phase $(70,90$, or $110 \mathrm{~km} / \mathrm{h})$. In the passive perception of various driving speed differences, the areas related to visual cognition and spatial attention such as temporal, occipital, parietal, frontal areas, and cerebellum were activated. As the driving speed difference increased, the number of activated voxels also increased in the areas related to visual cognition. However, the visual cognition related areas showed a different pattern from the spatial attention related area with an increase of the driving speed difference. This implies that each brain area has a different level of involvement in the passive perception of the driving speed difference, although both visual cognitions related areas and spatial attention related area are related to it.
\end{abstract}

Keywords: Driving speed difference, passive perception, visual cognition, spatial attention (vigilance)

\section{Introduction}

Driving is a complex task that requires a high level of cognitive processes such as perception, attention, decision making, and motor control. Among such perceptive and cognitive processes, visual cognition and spatial attention were known to be most deeply involved in driving performance [1].

Recently, functional Magnetic Resonance Imaging (fMRI) has been used for several studies on the

\footnotetext{
* Address for correspondence: Mi-Hyun Choi, Department of Biomedical Engineering, Research Institute of Biomedical Engineering, College of Biomedical \& Health Science, Konkuk University, Chungju, South Korea. Tel.: 82-43-840-3759; Fax: 82-43-851-0620; E-mail: mhchoi0311@gmail.com.
} 
change of neuronal activities relating to driving [2]. For example, the studies on the neuronal activities induced during a driving by visual cognition [3-6], spatial attention or vigilance $[3,5]$, and motor function [7] have been performed using fMRI. The study on the difference of visual cognition was also conducted for unfamiliar and familiar routes [8].

However, due to the requirement for an MRI compatibility, these studies had to be conducted in a simulated driving condition (e.g., a video game or a driving simulator etc) and, thus, it was hard to provide a realistic driving task for volunteers in practice. In other words, a simulated environment imposes a restraint on the correct evaluation of visual cognition, spatial attention, vigilance, and motor function in a real driving situation.

In addition, since the previous studies simply compared a rest condition with a driving condition [3, 5-7], or compared only two different driving speed cases, i,e., a low driving speed and a high driving speed $[3,6]$, the studies on neuronal activities relating to visual cognition and spatial attention have been rarely performed with various driving speeds.

In this study, the following three things were mainly considered to overcome the restrained aspects of the previous studies: First, a video recorded in a real driving situation on the road was used for a realistic visual stimulus. Second, for the observation of the neuronal activities induced by visual cognition and spatial attention of several driving speed differences, the driving video as a visual stimulation source was recorded with four different driving speeds such as $50,70,90$, and $110 \mathrm{~km} / \mathrm{h}$. Third, unlike the simple comparison of a rest condition with a driving condition in the previous studies, the driving speeds of 70,90 , and $110 \mathrm{~km} / \mathrm{h}$ were compared with a reference speed of $50 \mathrm{~km} / \mathrm{h}$ in this study and the passive perception of the driving speed differences was evaluated in terms of the neuronal activation area and the number of activated voxels.

\section{Methods}

\subsection{Subjects}

Eight healthy male (24.7 \pm 1.8 -years-of-age) and eight healthy female (22.2 \pm 1.7 -years-of-age) college students participated in the study. All the subjects had an experience of driving on a regular highway and their average driving career was $2.6 \pm 1.4$ years. They were also confirmed to be right handed by Edinburgh Handedness Inventory test [9]. None of the participants reported having a history of psychiatric or neurological disorders. The overall experimental procedure was explained to all subjects who provided their consent for the procedure. All examinations were performed under the regulation of our Institutional Review Committee.

\subsection{Experimental design}

A driving video as a stimulation source was recorded with four different driving speeds $(50,70,90$, and $110 \mathrm{~km} / \mathrm{h}$ ) in a real driving situation on a local rural road whose vehicular traffic was rare. The video recording was done using a VPC-SH1 camcorder (Sanyo, Tokyo, Japan) with a $1920 \times 1080$ pixels spatial resolution. The camcorder was fixed at the exact center of the dashboard inside a car in order to fully cover the frontal view of a car during the recording. The recording was repeated with each driving speed for a driving time of $1.5 \mathrm{~min}$. The recorded video was edited to $1 \mathrm{~min}$ in length. The edited video was played for the subjects through a $12 \times 15 \mathrm{~cm}^{2}$ LCD monitor attached to a head coil in a magnetic resonance (MR) system. 


\begin{tabular}{|c|c|c|c|c|c|}
\hline $\begin{array}{c}\text { Control } \\
\text { (Low speed) } \\
\text { (1min.) }\end{array}$ & $\begin{array}{c}\text { Stimulation } \\
\text { (High speed) } \\
\text { (1min.) }\end{array}$ & Control & Stimulation & Control & Stimulation \\
\hline $50 \mathrm{~km} / \mathrm{h}$ & $70 \mathrm{~km} / \mathrm{h}$ & $50 \mathrm{~km} / \mathrm{h}$ & $90 \mathrm{~km} / \mathrm{h}$ & $50 \mathrm{~km} / \mathrm{h}$ & $110 \mathrm{~km} / \mathrm{h}$ \\
\hline $50 \mathrm{~km} / \mathrm{h}$ & $70 \mathrm{~km} / \mathrm{h}$ & $50 \mathrm{~km} / \mathrm{h}$ & $110 \mathrm{~km} / \mathrm{h}$ & $50 \mathrm{~km} / \mathrm{h}$ & $90 \mathrm{~km} / \mathrm{h}$ \\
\hline $50 \mathrm{~km} / \mathrm{h}$ & $90 \mathrm{~km} / \mathrm{h}$ & $50 \mathrm{~km} / \mathrm{h}$ & $70 \mathrm{~km} / \mathrm{h}$ & $50 \mathrm{~km} / \mathrm{h}$ & $110 \mathrm{~km} / \mathrm{h}$ \\
\hline $50 \mathrm{~km} / \mathrm{h}$ & $90 \mathrm{~km} / \mathrm{h}$ & $50 \mathrm{~km} / \mathrm{h}$ & $110 \mathrm{~km} / \mathrm{h}$ & $50 \mathrm{~km} / \mathrm{h}$ & $70 \mathrm{~km} / \mathrm{h}$ \\
\hline $50 \mathrm{~km} / \mathrm{h}$ & $110 \mathrm{~km} / \mathrm{h}$ & $50 \mathrm{~km} / \mathrm{h}$ & $70 \mathrm{~km} / \mathrm{h}$ & $50 \mathrm{~km} / \mathrm{h}$ & $90 \mathrm{~km} / \mathrm{h}$ \\
\hline $50 \mathrm{~km} / \mathrm{h}$ & $110 \mathrm{~km} / \mathrm{h}$ & $50 \mathrm{~km} / \mathrm{h}$ & $90 \mathrm{~km} / \mathrm{h}$ & $50 \mathrm{~km} / \mathrm{h}$ & $70 \mathrm{~km} / \mathrm{h}$ \\
\hline
\end{tabular}

(a)

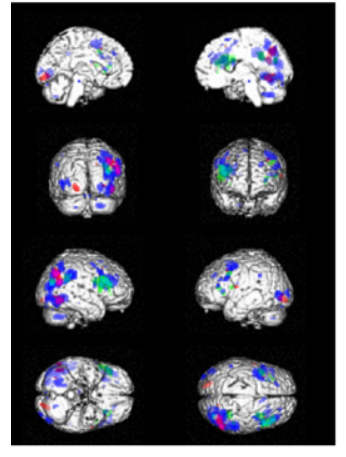

(b)

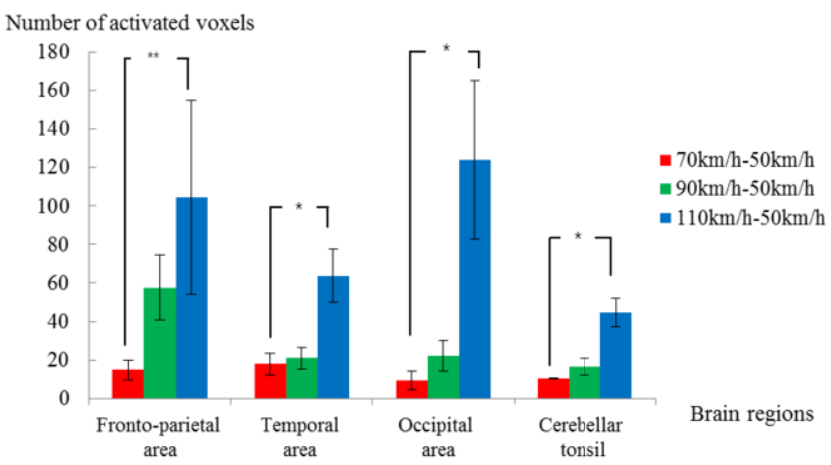

(c)

Fig. 1. (a) The stimulus protocol. (b) The brain activation areas under the conditions of $70-50 \mathrm{~km} / \mathrm{h}, 90-50 \mathrm{~km} / \mathrm{h}$, and $110-50$ $\mathrm{km} / \mathrm{h}$ (corrected $\mathrm{p}<0.05$ ). (c) The number of activated voxels in the fronto-parietal area, temporal area, occipital area, and cerebellar tonsil under the conditions of $70-50 \mathrm{~km} / \mathrm{h}, 90-50 \mathrm{~km} / \mathrm{h}$, and $110-50 \mathrm{~km} / \mathrm{h}$.

Each trial consisted of three blocks and each block included a 1 min control phase and a $1 \mathrm{~min}$ stimulation phase (Figure 1(a)). The video recorded with the lowest driving speed of $50 \mathrm{~km} / \mathrm{h}$ was shown in the control phase. In the stimulation phase, the videos recorded with the high driving speeds $(70,90$, or $110 \mathrm{~km} / \mathrm{h})$ were played. As illustrated in Figure 1(a), all the subjects participated in a total of six trials. The order of trials was counterbalanced and a $10 \mathrm{~min}$ break was provided between each trial.

After experiments, a subjective test was performed for evaluating whether all subjects correctly recognized the driving speed or not. In the subjective test, the volunteers were asked to choose one of the four driving speeds $(50,70,90$, and $110 \mathrm{~km} / \mathrm{h})$ in each phase when one of six trials shown in Figure 1(a) was arbitrarily selected and given to the subjects. As a result of the subjective test, there were six males ( $25.3 \pm 1.5$ years old) and four females $(21.8 \pm 2.2$ years old $)$ who correctly recognized all driving speeds in all phases, and the data of these 10 subjects were used for fMRI analysis.

\subsection{Imaging}

Imaging was conducted using a 3.0T ISOL Technology FORTE apparatus (ISOL Technology, Seoul, Korea) equipped with whole-body gradients and a quadrature head coil. Single-shot echo planar fMRI scans were acquired in 35 continuous slices, parallel to the anterior commissure-posterior commissure line. The parameters for fMRI were: repetition time/echo time $[\mathrm{TR} / \mathrm{TE}]=3,000 / 35 \mathrm{~ms}$, flip angle $=60$ degrees, field-of-view $(\mathrm{FOV})=240 \mathrm{~mm}$, matrix size $=64 \times 64$, slice thickness $=4 \mathrm{~mm}$, and in-plane resolution $=3.75 \mathrm{~mm}$. Five dummy scans in the beginning of each run were excluded to 
decrease the effect of non-steady-state longitudinal magnetization. T1-weighted anatomic images were obtained with a three-dimensional FLAIR sequence $(\mathrm{TR} / \mathrm{TE}=280 / 14 \mathrm{~ms}$, flip angle $=60$ degrees, FOV $=240 \mathrm{~mm}$, matrix size $=256 \times 256$, slice thickness $=4 \mathrm{~mm})$.

\section{4. fMRI data analysis}

The fMRI data were analyzed with SPM 8 software (Wellcome Department of Cognitive Neurology, London, UK). All functional images were aligned with the anatomic images of the study using affine transformation routines built into the SPM 8 program. The realigned scans were co-registered to the participant's anatomic images obtained within each session and normalized to a template image in SPM 8 that uses the space defined by the Montreal Neurologic Institute, which is very similar to Talairach and Tournoux's (1988) [10] stereotaxic atlas. Motion correction was works by lagging (shifting forward) the time-series data on each slice using sinc-interpolation. Time-series data were filtered with a 240 -s high-pass filter to remove artifacts due to cardiorespiratory and other cyclical influences. The functional map was smoothened with a 7-mm isotropic Gaussian kernel prior to statistical analysis [11-16]. Statistical analysis was done both individually and as a group using a general linear model and the theory of Gaussian random fields implemented in SPM 8. The brain areas activated by the passive perception of the driving speed difference between the reference speed of 50 $\mathrm{km} / \mathrm{h}$ and the test speeds of 70,90 , and $110 \mathrm{~km} / \mathrm{h}$ were extracted using a subtraction procedure and the number of voxels in these areas was calculated from the data of individual subjects as well as groups. Especially, not only the total number of voxels in all activated areas but also the number of activated voxels in each brain area of interest were calculated for each subject, and the analysis of one-way ANOVA (PASW 18.0) was performed for the evaluation of statistical significance. Also, Post-hoc test was used the bonferroni correction. The number of voxels in the frontal and parietal areas was calculated regarding these two areas as one, not separating each of them, because both of them are related to spatial attention or vigilance.

\section{Results}

The data from the group analysis of activation areas are summarized in Table 1 and Figure 1(b). The passive perception of the driving speed difference between $70 \mathrm{~km} / \mathrm{h}$ and $50 \mathrm{~km} / \mathrm{h}$ induced the activation of the right temporal fusiform gyrus, right superior temporal gyrus (BA 39), and right inferior parietal lobule (BA 39) areas. In the case of $90 \mathrm{~km} / \mathrm{h}$ and $50 \mathrm{~km} / \mathrm{h}$, the areas such as right frontal sub-gyral, bilateral middle frontal gyrus, right inferior frontal gyrus (BA 45), right parietal subgyral, and right middle temporal gyrus were activated. Lastly, when the driving speed of $110 \mathrm{~km} / \mathrm{h}$ was recognized in comparison to $50 \mathrm{~km} / \mathrm{h}$, the following areas were activated: right occipital fusiform gyrus, right temporal sub-gyral, right inferior parietal lobule, right superior frontal gyrus (BA 9), right frontal sub-gyral, bilateral middle frontal gyrus (right: BA 6, 8, left: BA 9, 46), right inferior frontal gyrus, left middle occipital gyrus (BA 18), left parietal supramarginal gyrus, and right cerebellar tonsil.

Figure 1(c) shows the number of activated voxels. In Figure 1(c), the fronto-parietal area includes right inferior parietal lobule, right frontal sub-gyral, bilateral middle frontal gyrus, right inferior frontal gyrus, right parietal sub-gyral, right superior frontal gyrus, and left parietal supramarginal gyrus. The temporal area contains right temporal fusiform gyrus, right superior temporal gyrus, right middle temporal gyrus, and right temporal sub-gyral. The occipital area comprises right occipital fusiform gyrus and left middle occipital gyrus. As the driving speed difference increased, the total number of 
Table 1

The MNI coordinates, $\mathrm{t}$-scores, and number of voxels in the activated areas by the subtraction method (70-50 km/h, 90-50 $\mathrm{km} / \mathrm{h}$, and $110-50 \mathrm{~km} / \mathrm{h})($ corrected $\mathrm{p}<0.05)$

\begin{tabular}{|c|c|c|c|c|c|c|}
\hline $\begin{array}{l}\text { Speed } \\
\text { difference }\end{array}$ & Num & t-sco & $\begin{array}{l}\text { MNI coord } \\
\mathrm{x}, \mathrm{y}, \mathrm{z}(\mathrm{mm}\end{array}$ & $\mathrm{Sid}$ & Region & $\begin{array}{l}\text { Brodmann } \\
\text { area }\end{array}$ \\
\hline $70 \mathrm{~km} / \mathrm{h}-50 \mathrm{~km} / \mathrm{h}$ & 26 & 6.25 & $48-60-12$ & $\mathrm{R}$ & Temporal Fusiform Gyrus & \\
\hline & 98 & 5.49 & $\begin{array}{lll}57 & -60 & 24\end{array}$ & $\mathrm{R}$ & Superior Temporal Gyrus & 39 \\
\hline & & 5.25 & $48-6639$ & $\mathrm{R}$ & Inferior Parietal Lobule & 39 \\
\hline $90 \mathrm{~km} / \mathrm{h}-50 \mathrm{~km} / \mathrm{h}$ & 191 & 5.35 & $33 \quad 6 \quad 24$ & $\mathrm{R}$ & Frontal Sub-Gyral & \\
\hline & & 5.16 & 423318 & $\mathrm{R}$ & Middle Frontal Gyrus & \\
\hline & & 4.94 & 512421 & $\mathrm{R}$ & Inferior Frontal Gyrus & 45 \\
\hline & 38 & 5.13 & $36-45 \quad 30$ & $\mathrm{R}$ & Parietal Sub-Gyral & \\
\hline & 21 & 4.66 & $39-7224$ & $\mathrm{R}$ & Middle Temporal Gyrus & \\
\hline & 27 & 4.55 & $\begin{array}{lll}-39 & 12 & 39\end{array}$ & $\mathrm{~L}$ & Middle Frontal Gyrus & \\
\hline $110 \mathrm{~km} / \mathrm{h}-50 \mathrm{~km} / \mathrm{h}$ & 227 & 6.25 & $42-69-12$ & $\mathrm{R}$ & Occipital Fusiform Gyrus & \\
\hline & 243 & 5.97 & $45-48-6$ & $\mathrm{R}$ & Temporal Sub-Gyral & \\
\hline & 232 & 5.86 & $54-33 \quad 33$ & $\mathrm{R}$ & Inferior Parietal Lobule & \\
\hline & 95 & 5.99 & 423630 & $\mathrm{R}$ & Superior Frontal Gyrus & 9 \\
\hline & 51 & 5.49 & 302124 & $\mathrm{R}$ & Frontal Sub-Gyral & \\
\hline & 10 & 5.1 & $-2724 \quad 6$ & $\mathrm{R}$ & Middle Frontal Gyrus & 6,8 \\
\hline & 6 & 4.51 & $-51-4536$ & $\mathrm{R}$ & Inferior Frontal Gyrus & \\
\hline & 18 & 4.44 & $\begin{array}{lll}48 & 15 & 18\end{array}$ & $\mathrm{R}$ & Inferior Frontal Gyrus & \\
\hline & 119 & 6.43 & $-36 \quad 951$ & $\mathrm{~L}$ & Middle Frontal Gyrus & 9,46 \\
\hline & 28 & 4.68 & $\begin{array}{lll}-51 & 15 & 33\end{array}$ & $\mathrm{~L}$ & Middle Occipital Gyrus & 18 \\
\hline & 11 & 4.62 & $\begin{array}{lll}-33 & -93 \quad 3\end{array}$ & $\mathrm{~L}$ & Parietal Supramarginal Gyrus & \\
\hline & 58 & 5.23 & $18-51-39$ & $\mathrm{RC}$ & Cerebellar Tonsil & \\
\hline
\end{tabular}

Note: R: right cerebral; L: left cerebral; RC: right cerebellar.

activated voxels also increased. According to the area-based analysis using one-way ANOVA, the number of activated voxels increased in the fronto-parietal area as the driving speed difference increased $(\mathrm{p}=.002)$. The number of activated voxels also increased in temporal $(\mathrm{p}=.013)$, occipital $(\mathrm{p}=.007)$, and cerebellar tonsil areas $(\mathrm{p}=.033)$ with an increasing driving speed difference. Interestingly, the $110 \mathrm{~km} / \mathrm{h}-50 \mathrm{~km} / \mathrm{h}$ trial showed an abrupt increase in the number of activated voxels in temporal, occipital, and cerebellar tonsil areas when compared to the $90 \mathrm{~km} / \mathrm{h}-50 \mathrm{~km} / \mathrm{h}$ and $70 \mathrm{~km} / \mathrm{h}-50 \mathrm{~km} / \mathrm{h}$ trials (Figure 1(c)). Bonferroni post-analysis verification revealed that the number of activated voxels in the $110 \mathrm{~km} / \mathrm{h}-50 \mathrm{~km} / \mathrm{h}$ trial compared to the $70 \mathrm{~km} / \mathrm{h}-50 \mathrm{~km} / \mathrm{h}$ trial increased with statistical significance in the fronto-parietal area $(\mathrm{p}=.002)$, temporal area $(\mathrm{p}=.052)$, occipital area $(\mathrm{p}=.018)$, and cerebellar tonsil $(\mathrm{p}=.050)$.

\section{Discussion}

An fMRI study was performed for the analysis of neuronal activation areas as well as the number of activate voxels induced by the passive perception of various driving speeds $(70,90$, and $110 \mathrm{~km} / \mathrm{h})$ in comparison to a reference speed of $50 \mathrm{~km} / \mathrm{h}$.

fMRI studies on the change of neuronal activations relevant to visual cognition and spatial attention during driving have been performed [17-21]. Brain areas as primary visual (V1) region, motionsensitive visual regions (V5/ area middle temporal: MT), and parietal cortex (Brodmann area 7) were 
reported as the main areas relating to visual cognition [17, 19], and the areas relevant to a high-order visual cognition were known to be cingulated, cerebellum, and occipital and parietal cortex areas [3]. On the other hand, it was reported that the areas relating to visual attention were occipital, inferiortemporal, and parahippocampal cortices, thalamus, and cerebellum $[18,21]$, and areas such as frontal and parietal cortical regions were related to spatial attention (or vigilance) [5]. Another fMRI study using a video game for car driving reported that the cognition of slow and high driving speeds not only activated such brain areas as occipital fusiform, cerebellum, middle and superior occipital lobes, inferior temporal lobe, and superior parietal lobe, which are related to a high-order visual cognition, but also activated the vigilance-related areas such as medial, inferior, middle, superior frontal lobes, and precuneus (parietal area) [3].

The results of our study on the passive perception of various driving speeds are consistent with the previous studies in that the areas related to visual cognition and spatial attention that were activated included the temporal, occipital, parietal, and frontal areas, as well as the cerebellum. As shown in Table 1 and Figure 1(b), it was especially observed in this study that the temporal and parietal areas were mainly activated when the difference in driving speed was small (e.g., $70 \mathrm{~km} / \mathrm{h}$ in comparison to $50 \mathrm{~km} / \mathrm{h}$ ). On the other hand, in the case of the cognition of a large driving speed difference (e.g., 90 $\mathrm{km} / \mathrm{h}$ or $110 \mathrm{~km} / \mathrm{h}$ compared to $50 \mathrm{~km} / \mathrm{h}$ ) neural activations occurred, especially in the frontal areas related to spatial attention or vigilance as well as the areas relevant to visual cognition $[3,18,21,22]$.

According to the previous studies done by Calhoun, et al. (2002) and Horikawa, et al. (2005), while the total number of voxels in all activation areas was larger in the driving condition than the rest condition, there was little difference in the total number of activated voxels between the cases of a low driving speed $(110-140 \mathrm{~km} / \mathrm{h})$ and a high driving speed $(160-224 \mathrm{~km} / \mathrm{h})$. In addition, the number of activated voxels increased at the high driving speed condition than the low driving speed condition in the areas such as medial, inferior, middle, superior frontal lobes, and precuneus, which are related to spatial attention (or vigilance), and these areas were regarded to be mainly involved in the cognition of the driving speed difference. However, occipital lobe and cerebellar areas related to a high-order visual cognition were reported as being irrelevant to the cognition of a driving speed because there was little difference in the number of activated voxels between the slow and fast driving conditions in spite of the occurrence of neuronal activations in both cases [3].

According to this study, as the driving speed difference increased, the number of activated voxels also tended to increase, which was not consistent with the previous studies. Furthermore, the brain area related to visual cognition seemed to be relevant to the passive perception of the driving speed difference because the neuronal activation appeared to be considerably increased in temporal area, occipital area, and cerebellum as well as fronto-parietal areas in the case of the $110 \mathrm{~km} / \mathrm{h}$ driving condition in comparison to $50 \mathrm{~km} / \mathrm{h}$. Orban and Orban, et al. reported that the discrimination of the speed of a randomly moving dot (70 stimuli $/ \mathrm{min}$ and $35 \mathrm{stimuli} / \mathrm{min})$ induced the neuronal activations in the areas such as superior temporal gyrus, lingual gyrus, and cuneus in occipital lobe. This result shows that the visual cognition areas are relevant to the cognition of the speed difference of a moving dot. Despite a different stimulation scheme, it can be inferred from these previous studies [20, 22, 23] and the present study that the areas of spatial attention (or vigilance) as well as visual cognition are related to the passive perception of driving speed difference. In the previous studies [3, 5-7], the neuronal activation patterns were induced in a single driving condition compared to a rest condition using a video game and applied only two diving speed (low and high speed). Unlike the previous studies, our study was performed with a realistic simulation scheme as a visual stimulus using a driving video recorded in a real driving situation on the road with various driving speeds. For such reasons, between our results and the previous studies were exhibited the discrepancy. Walter, et al. [24] 
reported that neuronal activations in brain areas such as MT and MST were reduced in the case of active driving (subjects steered the car themselves) when compared to the case of passive driving (a person outside the scanner was steering the car), which implies a difference between active driving and passive driving in terms of neuronal activation. Further studies will be thus required for the analysis of the difference of neuronal activations between active driving and passive driving.

Few studies have been performed for the analysis of the change of the number of voxels activated in each brain area due to the cognition of a various driving speed difference. According to our study, as the driving speed difference increased, the number of activated voxels also increased in the area related to spatial attention or vigilance such as a fronto-parietal area as well as the areas related to visual cognition such as temporal area, occipital area, and cerebellum. However, while the number of activated voxels in the area related to spatial attention or vigilance tended to increase in a linear manner, it did not in the areas related to visual cognition (i.e., it abruptly increased in the cognition of some driving speed differences, such as $110 \mathrm{~km} / \mathrm{h}$ compared to $50 \mathrm{~km} / \mathrm{h}$ ). This result implies that each brain area might be involved in the passive perception of the driving speed difference at a different level, which needs to be verified in a future study by increasing the number of subjects, offering a more various driving speed, and analyzing brain areas more in detail. A further study on functional connectivity using covariant analysis will also be desirable for a better understanding of the passive perception of the driving speed difference in brain areas.

Driving is very popular in daily life and it requires a complicated cognitive process. However, few fMRI studies have been performed so far because it is restricted in practice to deliver a simulated driving condition as real as possible in a MR environment. In this study, although the analysis of motor function was not made which is most important in driving, the change of neuronal activations was investigated due to the cognition of a various driving speed difference using a driving video recorded in a real driving situation on the road. It can be concluded that this study provides some basic data for elucidating the brain-function mechanism relating to a driving task based on a realistic visual stimulation.

\section{Acknowledgment}

This paper was supported by Konkuk University in 2015.

\section{References}

[1] J. Groeger, Understanding Driving, Psycholoy Press, East Sussex, UK, 2000, pp. 45-48.

[2] H.J. Spiers and E.A. Maguire, Neural substrates of driving behavior, NeuroImage 36 (2007), 245-255.

[3] V.D. Calhoun, J.J Pekar, V.B. McGinty, T. Adali, T.D. Watson and G.D. Pearlson, Different activation dynamics in multiple neural systems during simulated driving, Human Brain Mapping 16 (2002), 158-167.

[4] A. Campagne, T. Pebayle and A. Muzet, Correlation between driving errors and vigilance level: Influence of the driver's age, Physiological Behavior 80 (2004), 515-524.

[5] F.X. Graydon, R.A. Young, M.D. Tdss, R.J. Genik II, S. Posse, L. Hsieh and C. Green, Visual event detection during simulated driving: identifying the neural correlates with functional neuroimaging, Transportation Research Part F 7 (2004), 271-286.

[6] E. Horikawa, N. Okamura, M. Tashiro, Y. Sakurada, M. Maruyama, H. Arai, K. Yamaguchi, H. Sasaki, K. Yanai and M. Itoh, The neural correlates of driving performance identified using positron emission tomography, Brain and Cognition 58 (2005), 166-171.

[7] Y. Uchiyama, K. Ebe, A. Kozato, T. Okada and N. Sadato, The neural substrates of driving at a safe distance: A functional MRI study, Neuroscience Letters 352 (2003), 199-202. 
[8] M. Mader, A. Bresges, R. Topa, A. Busse, M. Forsting and E.R. Gizewski, Simulated car driving in fMRI-Cerebral activation patterns driving an unfamiliar and a familiar route, Neuroscience Letters 464 (2009), 222-227.

[9] R.C. Oldfield, The assessment and analysis of handedness, the Edinburgh inventory, Neuropsychologia 9 (1971), 97 113.

[10] J. Talairach and P. Tournoux, Co-Planar Stereotaxic Atlas of the Human Brain, Thieme Medical Pub, New York, Thieme, 1998, pp. 122.

[11] S.C. Chung, B. Lee, G.R. Tack, J.H. Yi, J.H. You and S.H. Son, The effect of oxygen administration on visuospatial cognitive performance: Time course data analysis of fMRI, International Journal of Neuroscience 16 (2006), 177-189.

[12] S.C. Chung, J.H. Sohn, B. Lee, G.R. Tack, J.H. Yi, J.H. You, J.H. Kwon, H.J. Kim and S.Y. Lee, A comparison of the mean signal change method and the voxel count method to evaluate the sensitivity of individual variability in visuospatial performance, Neuroscience Letters 418 (2007), 138-142.

[13] D.M. Lim, B.C. Min, H.J. Kim, M.H. Choi, S.J. Lee, J.H. Jun, B. Lee and S.C. Chung, Cerebral lateralization index based on intensity of bold signal of FMRI, International Journal of Neuroscience 118 (2008), 1628-1642.

[14] M.H. Choi, S.J. Lee, J.W. Yang, J.S. Choi, H.S. Kim, H.J. Kim, B.C. Min, S.J. Park, J.H. Jun, J.H. Yi, G.R. Tack and S.C. Chung, Activation of the limbic system under 30\% oxygen during a visuospatial task: An fMRI study, Neuroscience Letters 471 (2010), 70-73.

[15] H.S. Kim, M.H. Choi, H.J. Yoon, H.J. Kim, U.H. Jeong, S.J. Park, D.W. Lim, S.C. Chung and B. Lee, Cerebral activation and lateralization due to the cognition of a various driving speed difference: An fMRI study, Bio-Medical Materials and Engineering 24 (2014), 1133-1139.

[16] M. Mader, A. Bresges, R. Topal, A. Busse, M. Forsting and E.R. Gizewski, Simulated car driving in fMRI-Cerebral activation patterns driving an unfamiliar and a familiar route, Neuroscience Letters 464 (2009), 222-227.

[17] G. Allen, R.B Buxton, E.C. Wong and E. Courchesne, Attentional activation of the cerebellum independent of motor involvement, Science 275 (1997), 1940-1943.

[18] C.M. Arrington, T.H. Carr, A.R. Mayer and S.M. Rao, Neural mechanisms of visual attention: Object-based selection of a region in space, Journal of Cognitive Neuroscience 12 (2000), 106-117.

[19] K.J. Friston and C. Buchel, Attentional modulation of effective connectivity from V2 to V5/MT in humans, Proceedings of the National Academy of Sciences 97 (2000), 7591-7596.

[20] G.A. Orban, P. Dupont, B. De Bruyn, R. Vandenberghe, A. Rosier and L. Mortelmans, Human brain activity related to speed discrimination tasks, Experimental Brain Research 122 (1998), 9-22.

[21] D. Tomasi, T. Ernst, E.C. Caparelli and L. Chang, Practice-induced changes of brain function during visual attention: A parametric fMRI study at 4 Tesla, NeuroImage 23 (2004), 1414-1421.

[22] G.A. Orban, Visual processing in macaque area MT/V5 and its satellites (MSTd and MSTv), in: Extrastriate Cortex in Primates ,J.H. Kaas, K. Rockland, A. Peters, eds., Plenum Press, New York, 1997, pp. 359-434.

[23] G.A. Orban, P. Dupont, R. Vogels, B. De Bruyn, G. Bormans and L. Mortelmans, Task dependency of visual processing in the human visual system, Behavioral Brain Research 76 (1996), 215-223.

[24] H. Walter, S.C. Vetter, J. Grothe, A.P. Wunderlich, S. Hahn and M. Spitzer, The neural correlates of driving, NeuroReport 12 (2001), 1763-1767. 